\title{
The pragmatics of interlanguage
}

$\mathrm{R}$ esearch in pragmatics has evolved from its beginnings with different theoretical focuses, interfacing with cognition, communication and formal grammar, and thus making its definition quite difficult. Kasper (1996) defines the pragmatics of interlanguage as the "study of pragmatic knowledge in second language acquisition". O'Keeffe, Clancy, and Adolphs (2011) address pragmatics as the study of meaning interpretation. Perna, Goldnadel and Molsing (2016), admit that pragmatics has a phenomenological breadth that was sometimes considered undesirable. Nowadays, however, pragmatics finds itself in a very significant place within linguistics, because it presents an expressive amount of studies that are in external interface with Cognitive Psychology and Anthropology, in addition to presenting strict connections in its interface with Phonology, Morphology, Syntax and Semantics. The present edition of Letras de Hoje aims to show the breadth of research on themes related to the acquisition of pragmatic aspects in the context of additional language and $\mathrm{L} 1$.

The work of Ibaños and Costa presents the general aspects about the development of pragmatics, from its origins and strictly theoretical discussions, regarding pragmatics and its aspects. With this focus, the authors begin this journey from the classical period, which begins with the philosophers of logic and mind, and concludes it with the contemporary period, demonstrating that the variations around pragmatic concepts depend on the direction and the Interfaces that the various pragmatics pursue.

Strey and Monawar deal with linguistic-communicative competence in the additional language classroom. In order to support the theme, the authors discuss the symbolic mode, a phenomenon of natural language that occurs in the interface between form and use. First, they present the theoretical framework of Kratzer (1981, 1991, 2012) for the modality in natural language, in addition to the proposal of Yanovich $(2013 a, 2013 b)$ for the symbolic modality to then discuss the tools of cognitive pragmatics with the intention of analyzing the processing of language, especially in relation to procedural and conceptual meaning, based on Sperber and Wilson's (1995) theory of relevance.
In their article, Corsetti and Perna detail the results of a study of pragmatic markers with the methodology of corpus linguistics. The study involved quantitative and qualitative analyzes of a small specialized corpus, with the oral production of Brazilian English learners at CEFR level B1 and comparisons with reference corpora. The most common adverbs used to mediate speech segments, the explicit and implicit adverbial hedges used to mitigate representative speech acts, and the minimal response particles used by the interlocutor to express good receptivity were the elements analyzed. Subjects produced a limited number of speech markers and response particles.

Cruz and Lopez analyze the behavior of preintermediate English LE students who are preparing for the tourism industry in the transactional phase of a service encounter. In this specific context, they need to perform one of the tasks for which they are trained: giving information at a tourist visitor center. More specifically, this study analyzes (i) the strategies with which they provide tourist information, and (ii) how they address relational aspects during the meeting or manage relationships with tourists through those strategies and the information provided.

In her article, Sun seeks to answer whether positive transference of language exists through causative verbs in the Simultaneous Interpretation of ChinesePortuguese, and in what aspects such transfer occurs more frequently. The article begins by providing a brief discussion on linguistic transference and causative construction in Chinese and Portuguese. It goes to the methodological part, in which 10 discourses given by Chinese leaders in intercultural contexts and their simultaneous interpretations are transcribed and analyzed. The results show that both Chinese texts and Portuguese interpretations present a high percentage of causative verbs, and more than half of the data is interpreted through positive transference. It is found that positive transference exists and plays a very important role in the Simultaneous Interpretation of Chinese-Portuguese, and the referring strategy can help interpreters to facilitate the processing of the information to guarantee a better performance. 
Silva, Blanco and Blanco make considerations about the forms of treatment, specifically the personal pronouns of treatment in Brazilian Portuguese and Iberian Spanish, and present theories that approach the subject of courtesy. Based on data obtained from two Brazilian plays of the twentieth century, they analyze and compare the pragmatic strategies developed through the forms of treatment, both in Brazilian Portuguese and in Iberian Spanish. A second objective of the article, with didacticpedagogical motivation, was to offer teachers of foreign language some appreciations about the pragmalinguistic and sociopragmatic difficulties that, in the process of acquisition / learning of the forms of treatment, may occur through the influence of the mother tongue.

Similarly, Cohen is also concerned with the difficulties brought about by the distinction between pragmalinguistics and sociopragmatics in what concerns the acquisition of an L2 and seeks to describe how native and non-native L2 English teachers deal with these pragmatic issues in the classroom situation. The author presents an international research, in which he investigates the experiences of teachers of several languages with respect to the teaching of pragmatics. Then there are aspects related to pragmatic teaching, such as the background of the teacher, comparisons between second language teaching (L2) and foreign language (FL) and the potential of digital media and other means to provide models of pragmatic behavior.

Andrade and Rauen, in turn, present an article on teaching and learning Spanish as a FL. Their thesis is that humor facilitates the process and demonstrates that the use of relevance theory assists in understanding jokes. As an illustration, they analyze three jokes based on Yus' (2010) classification. They conclude that the application of the analytical apparatus of Sperber and Wilson's theory of relevance $(1986,1995)$ allows an empirical description of the ostensible-inferential processes necessary for the interpretation of jokes and that transferable jokes are more likely to be understood by students, when compared to substitutable and challenging jokes.

Goldnadel presents two complementary problems in pragmatics: the problem of the projection of presuppositions and the problem of the origin of the presuppositions. Although the descriptive problem of projection has received more attention, it cannot be dealt with without assuming some position on the explanatory problem, relative to the origin of the inferences considered presuppositional. The disagreement regarding the description of the projection of presuppositions contrasts, however, with a relatively historically constructed consensus on the nature of presuppositions. According to this consensus, presupposition is a phenomenon of a conventional nature, subject only in its projection to conversational order injunctions. More recently, this consensus has been shaken by a new wave of work, which in many ways has come to defend the idea that presupposition is a fundamentally conversational phenomenon. The author revisits some experiments that evaluate the way in which the processing of scalar implicatures and presuppositions takes place and tries to evaluate the importance, in the most general framework of this debate, of studies that investigate the way in which subjects in the process of acquisition process pragmatic inferences.

Preuss, Rodrigues and Oliveira Júnior expose the effects of explicit instruction on total and asseverative interrogative statements in Spanish as L2 and their possible interactions with individual variables such as attention capacity, frequency of use, linguistic and teaching experience. The authors present a quantitative study that covers linguistic tests of perception and production of statements, network test of attention and questionnaire of historical and linguistic proficiency. Their study has a convenience sample covering an experimental group that received explicit instruction on the target content in an intermediate period between the pre- and post-test language, and a control group that did not receive the instruction. The results show that there was no significant difference, related to the effect of the instruction.

Siqueira, Duarte Junior, Pereira, Ferrari and Lopes present an experimental research in the interface with cognitive linguistics. The authors conduct the study on the understanding of idiomatic expressions in the development of the language in Portuguese language. The purpose of this article is to present the application and results of a task of understanding idiomatic expressions in 270 participants in three age groups (children, adolescents and adults). The task consists of six items: sentences followed by an open question and a closed question. The comparison of the responses in the three groups indicates a gradual increase in the understanding of idiomatic expressions. Besides the age, the authors discuss the implication of the linguistic variables context, transparency, metaphoricity and familiarity in the understanding of the phenomenon. The analyzes suggest that this complex phenomenon must be studied taking into account all these aspects.

We would like to compliment all the authors who have enriched this edition of Letras de Hoje with their contributions. We hope that the topics addressed may stimulate further debates relevant for Pragmatic Studies, which may eventually contribute to the improvement and continued advancement of linguistics.

The Organizers 